\title{
Novel approaches to treating type 2 diabetes
}

\author{
Michael A. Nauck ${ }^{1}$ \\ Received: 20 October 2015 / Accepted: 9 November 2015 / Published online: 4 December 2015 \\ (C) Springer-Verlag Berlin Heidelberg 2015
}

Keywords Future therapy $\cdot$ Glucose-lowering therapy $\cdot$ New mechanisms $\cdot$ Pharmacotherapy $\cdot$ Type 2 diabetes

Today's armamentarium of medications suitable as a therapy for glucose-lowering in type 2 diabetes is far from perfect, both in terms of effectiveness (i.e. the ability to not only reduce, but normalise high glucose concentrations in a majority of patients) and safety/tolerability (i.e. freedom from adverse effects that might limit the dose or use of certain agents in some or even many patients). While we already have at least seven major drug classes approved and used for the treatment of type 2 diabetes, the search is on for more mechanisms that can be exploited for the treatment of hyperglycaemia or other aspects of diabetes mellitus. As part of the symposium on novel approaches to treating type 2 diabetes at the EASD 2015 meeting, Daniel Drucker, Toronto, Canada, introduced glucokinase activation and glucagon antagonism, while Fiona Gribble, Cambridge, UK, discussed G protein-coupled receptors (her mini-review is presented in a companion article [1]) as examples of novel avenues of attacking hyperglycaemia and obesity in patients with type 2 diabetes.

Glucokinase is a high $K_{\mathrm{m}}$ hexokinase specifically expressed in endocrine pancreatic beta cells and in hepatocytes that mediates the phosphorylation of glucose upon entry into cells. Its role in glucose metabolism can be understood based on the characterisation of gain-of- and loss-of-function mutations, which lead to hereditary hypoglycaemia or diabe-

Michael A. Nauck

michael.nauck@rub.de

1 Division of Diabetology, St. Josef-Hospital (Ruhr-University Bochum), Gudrunstr. 56, D-44791 Bochum, Germany tes, respectively [2]. In the liver, glucokinase enhances glucose uptake and storage/metabolism, whereas in the endocrine pancreas, it stimulates insulin secretion relative to ambient glucose concentrations. This may be partly welcome in type 2 diabetes, but may also carry the risk of hypoglycaemia, excess glucose uptake leading to VLDL triacylglycerol overproduction and fatty liver. A clinical study on a glucokinase activator reported that reductions in $\mathrm{HbA}_{1 \mathrm{c}}$ are substantial initially, but of weak durability, even for durations of up to only 30 weeks [3]. Hypoglycaemia was a frequent finding, including severe episodes. There was a small rise in mean body weight and some patients showed an increase in diastolic blood pressure - side effects which have to be considered undesirable. Thus, while glucokinase activation is a suitable target for lowering glucose in more general terms, lack of durability, a high risk for hypoglycaemia, and other undesired effects have prevented any compound taken into clinical development so far from getting anywhere near approval. Modified approaches will be needed to make glucokinase activation a success, if this is at all possible.

The role of hyperglucagonaemia in diabetes mellitus has been recognised for decades, and inhibition of the action of a hormone that causes hyperglycaemia is an obvious approach for the treatment of hyperglycaemia, especially considering the role of glucagon-stimulated hepatic glucose production for the induction and/or maintenance of hyperglycaemia, both in the fasting and post-meal state [4]. In animals, suppressing the expression of glucagon receptors reduces plasma glucose [5], and glucagon receptor knockout mice have improved oral glucose tolerance [6]. Clinical studies using glucagon antagonists such as MK-0893 [7] or LY29409021 [8] have demonstrated the potency of such an approach in terms of lowering $\mathrm{HbA}_{1 \mathrm{c}}$ (by up to $1.5 \%$ ); however, this is accompanied by elevations in liver enzymes, probably indicating steatosis, and by an elevation in LDL-cholesterol and apolipoprotein B. 
On the basis of animal studies, alpha cell hyperplasia and an increased risk of colon cancer need to be considered as possible consequences of long-term glucagon receptor blockade. Plasma concentrations of glucagon are elevated several-fold by glucagon receptor antagonism [9], as are plasma levels of GLP-1, possibly not secreted from L cells in the gut but, rather, from alpha cells in the endocrine pancreas. These cells do not normally produce GLP-1, but their pro-protein convertase pattern can be altered to allow generation of the peptide. Again in animal studies, glucagon receptor knockout leads to increased small and large bowel mass [10], mediated by GLP-1 and GLP-2 receptor signalling. Glucagon receptor signalling may be associated with adverse cardiac outcomes in certain animal models [11], and so glucagon receptor antagonism may be beneficial in this respect. Thus, although glucagon receptor inhibition has been shown to reduce glucose levels, the safety of and a positive risk-benefit relationship for this approach to treating hyperglycaemia in type 2 diabetes have not been established. Consequently, none of the compounds blocking the glucagon receptor is close to receiving approval or is being used in clinical practice.

While Daniel Drucker presented data specifically related to compounds that have been identified as glucokinase activators and glucagon antagonists, Fiona Gribble presented information on multiple G protein-coupled receptors (GPCRs). In the gastrointestinal tract, GPCRs are involved in stimulating the release of incretin hormones like GLP-1 in response to various nutrient stimuli; in the endocrine pancreas they are involved in insulin secretion from beta cells, and are involved in the regulation of appetite/food intake at the level of the central nervous system. The aim of this research group is not to study individual GPCRs or their ligands alone to define their role in pharmacologically modifying incretin hormone or insulin secretion or brain responses to gut-derived signals. Instead, they are using a systematic approach to identify GPCRs or their ligands that specifically stimulate, for example, GLP-1 release in specific regions of the gut, to identify receptors/ligands that stimulate insulin secretion without having similar effects on alpha cells (which would be counterintuitive if the intention is to lower glucose concentrations). Gribble's research group have developed promising tools to advance this field of science, and it is hoped that it may become possible to identify incretin secretion stimuli that act from the gut lumen (apical membrane) vs the circulation (basolateral membrane), from areas of the gut where the pattern of endocrine cells differs. Based on the effects of bariatric surgery, a hormonal pattern more similar to what the lower small intestines produce may be helpful to pharmacologically mimic the results of surgically induced weight loss without having to face the risks and irreversibility of these procedures.
Duality of interest MAN has been a member on advisory boards or has consulted with AstraZeneca, Boehringer Ingelheim, Eli Lilly \& Co., GlaxoSmithKline, Hoffman La Roche, Menarini/Berlin Chemie, Merck, Sharp \& Dohme, NovoNordisk and Versatis. He has received grant support from Eli Lilly \& Co., Menarini/Berlin-Chemie, Merck, Sharp \& Dohme, Novartis Pharma and Ypsomed. He has also served on the speakers' bureau of AstraZeneca, Boehringer Ingelheim, Eli Lilly \& Co., Hoffman La Roche, Menarini/Berlin Chemie, Merck, Sharp \& Dohme and NovoNordisk.

Contribution statement The author was the sole contributor to this paper.

\section{References}

1. Reimann F, Gribble FM (2015) G protein-coupled receptors as new therapeutic targets for type 2 diabetes. Diabetologia doi:10.1007/ s00125-015-3825-Z

2. Gidh-Jain M, Takeda J, Xu LZ et al (1993) Glucokinase mutations associated with non-insulin-dependent (type 2) diabetes mellitus have decreased enzymatic activity: implications for structure/function relationships. Proc Natl Acad Sci U S A 90:1932-1936

3. Meininger GE, Scott R, Alba M et al (2011) Effects of MK-0941, a novel glucokinase activator, on glycemic control in insulin-treated patients with type 2 diabetes. Diabetes Care 34:2560-2566

4. Lee Y, Berglund ED, Wang MY et al (2012) Metabolic manifestations of insulin deficiency do not occur without glucagon action. Proc Natl Acad Sci U S A 109:14972-14976

5. Sloop KW, Cao JX, Siesky AM et al (2004) Hepatic and glucagon-like peptide-1-mediated reversal of diabetes by glucagon receptor antisense oligonucleotide inhibitors. J Clin Invest 113:1571-1581

6. Gelling RW, Du XQ, Dichmann DS et al (2003) Lower blood glucose, hyperglucagonemia, and pancreatic alpha cell hyperplasia in glucagon receptor knockout mice. Proc Natl Acad Sci U S A 100: 1438-1443

7. Engel SS, Xu L, Andryuk PJ et al (2011) Efficacy and tolerability of MK-0893, a glucagon receptor antagonist (GRA), in patients with type 2 diabetes (T2DM). Diabetes 60(Suppl 1): 309-OR. Available from http://professional. diabetes.org/Abstracts Display.aspx?TYP $=1 \& C I D=86894$, accessed 18 Nov 2015 (Abstract)

8. Xiong Y, Guo J, Candelore MR et al (2012) Discovery of a novel glucagon receptor antagonist $N-[(4-\{(1 S)-1-[3-(3,5-$ dichlorophenyl)-5-(6-methoxynaphthalen-2-yl)-1H-pyrazol-1yl]ethyl phenyl)carbonyl]- $\beta$-alanine (MK-0893) for the treatment of type II diabetes. J Med Chem 55:6137-6148

9. Longuet C, Sinclair EM, Maida A et al (2008) The glucagon receptor is required for the adaptive metabolic response to fasting. Cell Metab 8:359-371

10. Koehler JA, Baggio LL, Yusta B et al (2015) GLP-1R agonists promote normal and neoplastic intestinal growth through mechanisms requiring Fgf7. Cell Metab 21:379-391

11. Ali S, Ussher JR, Baggio LL et al (2015) Cardiomyocyte glucagon receptor signaling modulates outcomes in mice with experimental myocardial infarction. Mol Metab 4:132-143 